\title{
THE A-MODEL WITH MUTUALLY EQUAL MODEL PARAMETERS CAN LEAD TO A HILBERT SPACE MODEL
}

\author{
RYTIS JURŠĖNAS
}

\begin{abstract}
It is known that the A-model for higher order singular perturbations can be considered as a Hilbert space model if the model parameters are mutually distinct, and that it is necessarily a Pontryagin space model if otherwise. In this note we demonstrate that the A-model with mutually equal model parameters can nonetheless lead to a Hilbert space model if the extensions in the model space are instead described by suitable linear relations.
\end{abstract}

Mathematics subject classification (2020): 47A56, 47B25, 47B50, 35P05.

Keywords and phrases: Finite rank higher order singular perturbation, cascade (A) model, Hilbert space, scale of Hilbert spaces, Pontryagin space, ordinary boundary triple, Krein $Q$-function, Weyl function, gamma field, symmetric linear relation, proper extension, resolvent.

\section{REFERENCES}

[1] T. AzIzov AND I. IoKHVIDov, Linear Operators in Spaces with an Indefinite Metric, John Wiley \& Sons. Inc., 1989.

[2] J. Behrndt, V. A. Derkach, S. Hassi, AND H. DE SNOO, A realization theorem for generalized Nevanlinna families, Operators and Matrices, 5 (4): 679-706, 2011.

[3] J. BEHRNDT, S. HASSI, AND H. DE SNOO, Boundary relations, unitary colligations, and functional models, Compl. Anal. Oper. Theory, 3 (1): 57-98, 2009.

[4] J. Behrndt, S. Hassi, And H. De Snoo, Boundary Value Problems, Weyl Functions, and Differential Operators, chapter 2, Boundary Triplets and Weyl Functions, pages 107-167, Birkhauser, 2020.

[5] J. Behrndt, A. Luger, AND C. TRUnK, On the negative squares of a class of self-adjoint extensions in Krein spaces, Math. Nachr., 286 (2-3): 118-148, 2013.

[6] K. Daho AND H. Langer, Matrix functions of the class $N_{K}$, Math. Nachr., 120 (1): 275-294, 1985.

[7] V. DERKACH, On generalized resolvents of Hermitian operators in Krein spaces, Ukrainian Math. J., 46 (9): 1248-1262, 1994.

[8] V. DERKACH, On Weyl function and generalized resolvents of a Hermitian operator in a Krein space, Integr. Equ. Oper. Theory, 23 (4): 387-415, 1995.

[9] V. DERKACH, Abstract interpolation problem in Nevanlinna classes, Modern Analysis and Applications, 190: 197-236, 2009.

[10] V. DeRKACH, Boundary triplets, Weyl functions, and the Krein formula, volume 1-2 of Operator Theory, chapter 10, pages 183-218, Springer, Basel, 2015.

[11] V. Derkach, S. Hassi, AND H. DE SnOo, Singular perturbations of self-adjoint operators, Math. Phys. Anal. Geom., 6 (4): 349-384, 2003.

[12] V. Derkach, S. Hassi, M. Malamud, And H. DE Snoo, Boundary relations and their Weyl families, Trans. Amer. Math. Soc., 358 (12): 5351-5400, 2006.

[13] V. Derkach, S. Hassi, M. Malamud, And H. De Snoo, Boundary relations and generalized resolvents of symmetric operators, Russ. J. Math. Phys., 16 (1): 17-60, 2009.

[14] V. A. Derkach And M. M. Malamud, Generalized Resolvents and the Boundary Value Problems for Hermitian Operators with Gaps, J. Func. Anal., 95 (1): 1-95, 1991. 
[15] Vladimir Derkach, Seppo Hassi, and Mark Malamud, Generalized boundary triples, I. Some classes of isometric and unitary boundary pairs and realization problems for subclasses of Nevanlinna functions, Mathematische Nachrichten, 293 (7): 1278-1327, 2020.

[16] Vladimir Derkach, Seppo Hassi, Mark Malamud, and Henk de Snoo, Boundary triplets and Weyl functions. Recent developments, In Seppo Hassi, Hendrik S. V. de Snoo, and Franciszek Hugon Szafraniec, editors, Operator Methods for Boundary Value Problems, London Math. Soc. Lecture Note Series, volume 404, chapter 7, pages 161-220. Cambridge University Press, UK, 2012.

[17] A. Dijksma, P. Kurasov, And Yu. Shondin, High Order Singular Rank One Perturbations of a Positive Operator, Integr. Equ. Oper. Theory, 53: 209-245, 2005.

[18] A. DiJKsma AND H. LANGER, Compressions of self-adjoint extensions of a symmetric operator and M. G. Krein's resolvent formula, Integr. Equ. Oper. Theory, 90 (41): 1-30, 2018.

[19] S. Hassi, H. S. V. De Snoo, And F. H. Szafraniec, Componentwise and Cartesian decompositions of linear relations, Dissertationes Mathematicae, 465: 1-59, 2009.

[20] S. HASSi, M. MALAmUd, AND V. MogiLeVsKiI, Unitary equivalence of proper extensions of a symmetric operator and the Weyl function, Integr. Equ. Oper. Theory, 77 (4): 449-487, 2013.

[21] S. Hassi, Z. Sebestyén, H. S. V. De Snoo, and F. H. SZafraniec, A canonical decomposition for linear operators and linear relations, Acta Math. Hungar., 115 (4): 281-307, 2007.

[22] R. JURŠĖNAS, The peak model for the triplet extensions and their transformations to the reference Hilbert space in the case of finite defect numbers, arXiv:1810.07416v2, 2020.

[23] RYTIS JURŠĖNAS, On some extensions of the A-model, Opuscula Mathematica, 40 (5): 569-597, 2020.

[24] H. Langer And B. Textorius, On generalized resolvents and Q-functions of symmetric linear relations (subspaces) in Hilbert space, Pacific. J. Math., 72 (1): 135-165, 1977. 